\title{
Investigação no contexto brasileiro sobre gestão do conhecimento/aprendizagem/tecnologia de informação: pesquisa realizada na Scientific Electronic Library Online
}

\author{
Research in Brazilian Context about Knowledge Management / Learning / \\ Information Technology: Search Conducted in Scientific Electronic Library Online
}

\author{
Wagner Igarashi ${ }^{1}$ \\ Deisy Cristina Corrêa Igarashi \\ Eleonora Milano Falcão Vieira ${ }^{3}$ \\ José Leomar Todesco ${ }^{4}$
}

\begin{abstract}
Resumo
Este artigo investiga a produção cientifica sobre gestão do conhecimento/aprendizagem/tecnologia de informação por meio de pesquisa na Scientific Electronic Library Online. Primeiro de tudo, foram encontrados 194 artigos que abordavam esses termos separadamente. Num segundo momento, dos 194 artigos foram selecionados 49 que tratavam da gestão do conhecimento organizacional, enfocando o processo e o nível de aprendizagem e utilizando ferramentas de tecnologia de informação. Nestes 49 artigos foi aplicada a classificação de aprendizagem proposta por Pawlowsky, Forslin e Reinhardt (2003), o que possibilitou selecionar oito artigos que apresentavam um maior número de ocorrências relativas $\dot{a}$ classificação. Por fim, esses oito artigos foram mapeados, constatando-se que a gestão do conhecimento organizacional se refere a um processo de mudança cultural, e que a aprendizagem é um elemento essencial a ser apoiado por ferramentas de tecnologia de informação. Essa constatação possibilitou ainda o entendimento de que (i) a tecnologia de informação pode auxiliar na gestão do conhecimento como um todo (alavancando, conduzindo e direcionando), e (ii) de que é necessário adaptar a tecnologia de informação ao contexto organizacional.
\end{abstract}

Palavras-chave: gestão do conhecimento; aprendizagem; tecnologia da informação.

Abstract

This article investigates the scientific production of knowledge management/learning/technology of information, through search carried out in the Scientific Electronic Library Online. Initially, we analyzed 194 articles that discuss the terms individually and selected 49 articles that addressed the management of organizational knowledge, focusing on the process and the level of organizational learning and the use of information technology tools. In these 49 articles, the classification proposed by Pawlowsky et al. (2003) about organizational learning has been applied. This procedure allowed the selection of 8 articles, which had a higher number of incidents relating to the classification. Finally, these 8 articles were mapped and, as a result, it was found that the perception that the organizational knowledge management refers to a process of cultural change and that learning is a key element to be supported by information technology tools has been confirmed. These conclusions allowed the following understandings: i) the possibility of information technology assists in the management of knowledge as a whole (lever, leads and directs), and (ii) the need to adapt the information technology to the organizational context.

Key words: knowledge management; learning; information technology.

\footnotetext{
${ }^{1}$ Doutorando em Engenharia e Gestão do Conhecimento pela Universidade Federal de Santa Catarina. Docente na Universidade Estadual do Oeste do Paraná. Endereço: Rua Belarmino de Mendonça, 880 - Bloco B ap 111 - Centro - Foz do Iguaçu/Paraná - Brasil - CEP: 85851100 - E-mail: wigarash@gmail.com

${ }^{2}$ Doutorando em Engenharia de Produção pela Universidade Federal de Santa Catarina. Docente da Universidade Estadual do Oeste do Paraná

Endereço: Rua Belarmino de Mendonça, 880 - Bloco B ap 111 - Centro - Foz do Iguaçu/Paraná - Brasil - CEP: 85851100 - E-mail: deisyigarashi@gmail.com

${ }^{3}$ Doutoranda em Engenharia e Gestão do Conhecimento na Universidade Federal de Santa Catarina - UFSC. Professora do Departamento de Ciências Contábeis da Universidade Federal de Santa Catarina. Endereço: Rua Almirante Barroso, 22 apto 402 - João Paulo - Florianópolis/Santa Catarina - Brasil - CEP: 88030460 - E-mail: eleonorafalcao@gmail.com

${ }^{4}$ Doutor em Engenharia de Produção. Docente na Universidade Federal de Santa Catarina. Endereço: Rua Prof. Ayrton Roberto de Oliveira, 32, $7^{\circ}$ andar Itacorubi - Florianópolis/Santa Catarina - Brasil - CEP: 88034050 - E-mail: tite@stela.org.br
} 
Investigação no contexto brasileiro sobre gestão do conhecimento/aprendizagem/tecnologia de informação: pesquisa realizada na Scientific Electronic Library Online
Wagner Igarashi

Deisy Cristina Corrêa Igarashi

Eleonora Milano Falcão Vieira José Leomar Todesco

\section{Introdução}

A partir da revista aos estudos desenvolvidos por Bitencourt (2005), Fialho et al (2006), Leite e Porsse (2005) e Ruas (2005) acerca de gestão do conhecimento, os autores deste artigo verificaram que

a gestão do conhecimento organizacional refere-se a um processo de mudança cultural, sendo a aprendizagem um elemento essencial, que deveria ser apoiado por ferramentas de tecnologia de informação.

Salientando essa relação entre gestão do conhecimento/aprendizagem/tecnologia de informação, Fialho et al (2006, p.5) observa que

as idéias sobre a Gestão do Conhecimento e aprendizagem nas organizações são, na verdade, conseqüência da evolução do pensamento humano, das tecnologias de informação e comunicação, da gestão de pessoas e da própria Gestão do Conhecimento.

Dada a importância dessa questão e a necessidade de se conhecer como as diversas áreas de conhecimento no contexto nacional percebem esse tema, este estudo visa apresentar um panorama da produção científica brasileira sobre gestão do conhecimento/aprendizagem/tecnologia de informação. Para tanto, procedeu-se à análise bibliométrica nos trabalhos publicados na biblioteca eletrônica Scientific Electronic Library Online (Scielo).

Este estudo é segmentado em três momentos. Inicialmente, são focados os trabalhos que abordam separadamente os termos aprendizagem, gestão do conhecimento e tecnologia de informação, com vistas à identificação dos periódicos e das respectivas áreas de conhecimento nos quais os artigos são publicados. Na seqüência, investigaram-se os periódicos que mais publicam sobre o tema, a existência (ou não) da(s) instituição(ões) que se configura(m) como benchmarking nessa área de pesquisa, a quantidade de autores/coautores participantes da elaboração do artigo publicado, a existência (ou não) de pesquisadores brasileiros com trajetória estabelecida na área e a existência ou não de interação entre as instituições quanto à publicação conjunta. A partir da identificação das publicações científicas que tratam do tema, foram selecionadas aquelas que enfocam a gestão do conhecimento organizacional, considerando-se aspectos quanto à aprendizagem organizacional, que, simultaneamente, fazem uso de ferramentas advindas da tecnologia de informação. Nesta etapa é realizado o mapeamento desses artigos, buscando-se evidenciar como a tecnologia de informação tem auxiliado na aprendizagem organizacional e na gestão do conhecimento, gerando, assim, reflexão sobre o tema.

Para a seleção desses artigos, foi adotada a categorização proposta por Pawlowsky, Forslin e Reinhardt (2003) quanto à aprendizagem organizacional, em relação a dois enfoques. $\mathrm{O}$ primeiro enfoque, em relação aos processos de aprendizagem organizacional (difusão, integração, criação, ação e identificação) e o segundo enfoque, quanto ao nível de aprendizagem organizacional (individual, grupo, organizacional e interorganizacional). Para a amostragem, foram selecionados os artigos que apresentaram maior número de ocorrência dos mesmos, considerando como fator excludente nessa seleção, a não ocorrência de um dos elementos, seja em relação ao processo de aprendizagem, seja em relação ao nível de aprendizagem.

Além desta introdução, este artigo apresenta, na próxima seção, a metodologia da pesquisa, mostrando o enquadramento metodológico, a ferramenta de intervenção empregada, a população e amostra selecionadas, além dos procedimentos para coleta e análise dos dados. A seção seguinte traz a análise desses dados e os indicadores identificados por meio do estudo bibliométrico. Em seguida, são formuladas as considerações finais e feitas recomendações para futuras pesquisas, levando-se em conta as limitações deste estudo. Por último, constam as referências bibliográficas e os sites consultados na pesquisa. 
Investigação no contexto brasileiro sobre gestão do conhecimento/aprendizagem/tecnologia de informação: pesquisa realizada na Scientific Electronic Library Online
Wagner Igarashi

Deisy Cristina Corrêa Igarashi

Eleonora Milano Falcão Vieira José Leomar Todesco

\section{Enquadramento metodológico e os procedimentos para coleta e análise dos dados}

Nesta pesquisa, de caráter empírico-descritiva, a análise bibliométrica é o instrumento de intervenção utilizado para o levantamento de indicadores relativos à produção científica na área de gestão do conhecimento/aprendizagem/tecnologia de informação.

Para o desenvolvimento do estudo, adota-se a noção de bibliometria oferecida por Macias-Chapula (1998, p.134) de que este se refere ao "estudo dos aspectos quantitativos da produção, disseminação e uso da informação registrada". Caldas e Tinoco (2003, p.102) apresentam cinco tipos de métodos utilizados pela bibliometria: análise de citações, análise de co-citação, agrupamento bibliográfico, co-word anlaysis e webmetria. Este estudo utiliza a análise de citações e o agrupamento bibliográfico. Busca-se a medida quantitativa das publicações científicas de pesquisadores individuais e/ou instituições, considerando-se como base, periódicos com seleção arbitrada e a medida qualitativa dessas publicações, por meio de indicadores que incluem estudos comparativos de publicações e citações (PRITCHARD, 1998).

Esta investigação teve como fonte de coleta de dados os artigos científicos publicados na biblioteca eletrônica Scientific Electronic Library Online (Scielo). Para a seleção da amostra referente aos aspectos macro, foram realizadas buscas na Scielo, a partir da categoria artigo, por meio do índice de assuntos, na qual se pesquisou os termos "aprendizagem", "gestão do conhecimento" e "tecnologia de informação".

Salienta-se que a Scielo foi escolhida como fonte de busca por ser uma "biblioteca eletrônica que abrange uma coleção selecionada de periódicos científicos brasileiros" (SCIELO, 2007). O critério para a seleção foi o de relação direta ou indireta com os termos em, pelo menos, uma das seguintes seções: "Título dos artigos", "Palavras-chave dos artigos" ou "Resumo do artigo".

A delimitação da amostra referente ao segundo momento da pesquisa foi realizada por meio da leitura dos resumos dos 194 artigos selecionados, com vistas a identificar quais desses artigos efetivamente faziam uso da tecnologia de informação. Essa análise gerou a seleção de uma amostragem de 49 artigos. A partir daí, foi possível fazer o recorte para o terceiro momento; qual seja, investigar a tendência desses textos, buscando verificar as ferramentas de tecnologia de informação utilizadas no âmbito organizacional no processo de gestão do conhecimento e aprendizagem organizacional.

A seleção dessa fonte caracteriza-se como intencional e não-probabilística. Assim, os resultados da investigação apresentados permitem inferir tendências e perspectivas, não sendo possível generalizações de caráter incisivo. Entretanto, acredita-se estar contribuindo com esse mapeamento para a pesquisa na área de gestão do conhecimento/aprendizagem/tecnologia de informação, a qual pode ser expandida em futuros trabalhos.

Uma vez apresentado o enquadramento metodológico e os procedimentos para coleta e análise dos dados, passa-se a apresentação dos mesmos.

\section{Apresentação e análise dos dados}

A investigação e a apresentação dos indicadores bibliométricos sobre a produção científica da gestão do conhecimento/aprendizagem/tecnologia de informação será apresentada, seqüencialmente, nos três momentos mencionados.

\section{Pesquisa no sentido macro}

No que se refere ao primeiro momento da pesquisa, a investigação dos artigos científicos publicados na Scielo objetivou identificar quais os periódicos e as áreas de conhecimento em que os artigos foram publicados, a partir da busca dos termos supracitados na "lista por assunto" da biblioteca. Essa seleção resultou na 
Investigação no contexto brasileiro sobre gestão do conhecimento/aprendizagem/tecnologia de informação: pesquisa realizada na Scientific Electronic Library Online
Wagner Igarashi

Deisy Cristina Corrêa Igarashi

Eleonora Milano Falcão Vieira José Leomar Todesco

identificação e seleção dos periódicos que se dedicaram ao tema, bem como do ano de publicação dos mesmos, o que é apresentado na tabela 1.

Tabela 1

Número de artigos publicados sobre aprendizagem/ gestão do conhecimento/tecnologia de informação ao ano

\begin{tabular}{|c|c|c|c|c|c|c|c|c|c|c|c|c|c|c|c|c|c|}
\hline \multirow{2}{*}{ Periódicos que se dedicaram ao tema } & \multicolumn{7}{|c|}{1900} & \multicolumn{8}{|c|}{2000} & \multirow{2}{*}{ Total } & \multirow{2}{*}{$\%$} \\
\hline & 77 & 78 & 81 & 92 & 97 & 98 & 99 & $\mathbf{0}$ & 1 & 2 & 3 & 4 & 5 & 6 & 7 & & \\
\hline Psicologia: Reflexão e Crítica & & & & & & & 4 & 3 & 2 & & 2 & 3 & & 3 & & 17 & $9 \%$ \\
\hline Ciência da Informação & & & & & & & 2 & & 1 & 4 & 2 & 1 & 1 & 1 & & 12 & $6 \%$ \\
\hline $\begin{array}{l}\text { Revista Latino-Americana de } \\
\text { Enfermagem }\end{array}$ & & & & & & 2 & 1 & 2 & & 1 & 4 & & & 2 & & 12 & $6 \%$ \\
\hline ERA eletrônica & & & & & & & & & & & & 8 & 1 & 2 & & 11 & $6 \%$ \\
\hline Psicologia em Estudo & & & & & & & & & 1 & & 1 & 3 & 1 & 4 & & 10 & $5 \%$ \\
\hline Arquivos de Neuropsiquiatria & & & & & & & & 2 & & & & 2 & 2 & 3 & & 9 & $5 \%$ \\
\hline Psicologia: Teoria e Pesquisa & & & & & & & & 1 & 3 & & 1 & & 3 & 1 & & 9 & $5 \%$ \\
\hline Produção & & & & & & & & & & & 4 & & 3 & 1 & & 8 & $4 \%$ \\
\hline Ciência \& Saúde Coletiva & & & & & 1 & 1 & & 1 & 1 & & 1 & & 1 & & 1 & 7 & $4 \%$ \\
\hline Estudos de Psicologia (Natal) & & & & & & & & & 1 & 2 & 1 & & & 2 & & 6 & $3 \%$ \\
\hline Gestão \& Produção & & & & & & & & & & 1 & 2 & 1 & 1 & 1 & & 6 & $3 \%$ \\
\hline Cadernos Cedes & & & & & & & & & & & 1 & & 4 & & & 5 & $3 \%$ \\
\hline Cadernos de Pesquisa & & & & & & & & & 1 & 1 & 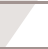 & & 1 & 2 & & 5 & $3 \%$ \\
\hline $\begin{array}{l}\text { Ensaio: Avaliação e Políticas Públicas em } \\
\text { Educação }\end{array}$ & & & & & & & & & & & & & 4 & 1 & & 5 & $3 \%$ \\
\hline $\begin{array}{l}\text { Interface - Comunicação, Saúde, } \\
\text { Educação }\end{array}$ & & & & & & & & & & & & & 2 & 3 & & 5 & $3 \%$ \\
\hline Revista Brasileira de Ensino de Física & & & & & & & & & & & & 2 & 1 & 2 & & 5 & $3 \%$ \\
\hline Revista de Saúde Pública & 1 & 1 & 1 & 1 & & & & & & & & & 1 & & & 5 & $3 \%$ \\
\hline Anais da Academia Brasileira de Ciências & & & & 8 & & & & & & & & 4 & & & & 4 & $2 \%$ \\
\hline Revista Brasileira de Educação Médica & & & & T & & & & & & & & 1 & & 3 & & 4 & $2 \%$ \\
\hline São Paulo em Perspectiva & & & & & & & & 3 & & 1 & & & & & & 4 & $2 \%$ \\
\hline Educação \& Sociedade & & & & & & & & 1 & & & & & 2 & & & 3 & $2 \%$ \\
\hline Educação e Pesquisa & & & & & & & & & & 1 & 1 & & & 1 & & 3 & $2 \%$ \\
\hline História, Ciências, Saúde-Manguinhos & & & & & & & & & & & & & 3 & & & 3 & $2 \%$ \\
\hline Perspectivas em Ciência da Informação & & & & 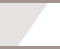 & & & & & & & & & & 3 & & 3 & $2 \%$ \\
\hline $\begin{array}{l}\text { Pró-Fono Revista de Atualização } \\
\text { Científica }\end{array}$ & & & & & & & & & & & & & 3 & & & 3 & $2 \%$ \\
\hline Jornal de Pediatria & & & & & & & & & & & & 1 & & 1 & & 2 & $1 \%$ \\
\hline Psicologia \& Sociedade & & & & & & & & & & & & 2 & & & & 2 & $1 \%$ \\
\hline Revista Brasileira de Educação & & & & & & & & & & & 1 & 1 & & & & 2 & $1 \%$ \\
\hline Revista Brasileira de Educação Especial & & & & & & & & & & & & & 1 & 1 & & 2 & $1 \%$ \\
\hline Revista Brasileira de Otorrinolaringologia & & & & & & & & & & & & 1 & 1 & & & 2 & $1 \%$ \\
\hline Revista Brasileira de Psiquiatria & & & & & & & & & & & & & 1 & & 1 & 2 & $1 \%$ \\
\hline $\begin{array}{l}\text { Sba: Controle \& Automação Sociedade } \\
\text { Brasileira de Automática }\end{array}$ & & & & & & & & & & 1 & & 1 & & & & 2 & $1 \%$ \\
\hline $\begin{array}{l}\text { Arquivos Brasileiros de Endocrinologia } \\
\text { \& Metabologia }\end{array}$ & & & & & & & & & & & & 1 & & & & 1 & $1 \%$ \\
\hline Arquivos Brasileiros de Oftalmologia & & & & & & & & & & & 1 & & & & & 1 & $1 \%$ \\
\hline Cadernos de Saúde Pública & & & & 1 & & & & & & & & & & & & 1 & $1 \%$ \\
\hline $\begin{array}{l}\text { DELTA: Documentação de Estudos em } \\
\text { Lingüística Teórica e Aplicada }\end{array}$ & & & & & & & & & & & & & 1 & & & 1 & $1 \%$ \\
\hline Estudos Avançados & & & & & & & & & & & & & & 1 & & 1 & $1 \%$ \\
\hline
\end{tabular}


Investigação no contexto brasileiro sobre gestão do conhecimento/aprendizagem/tecnologia de informação: pesquisa realizada na Scientific Electronic Library Online
Wagner Igarashi Deisy Cristina Corrêa Igarashi Eleonora Milano Falcão Vieira José Leomar Todesco

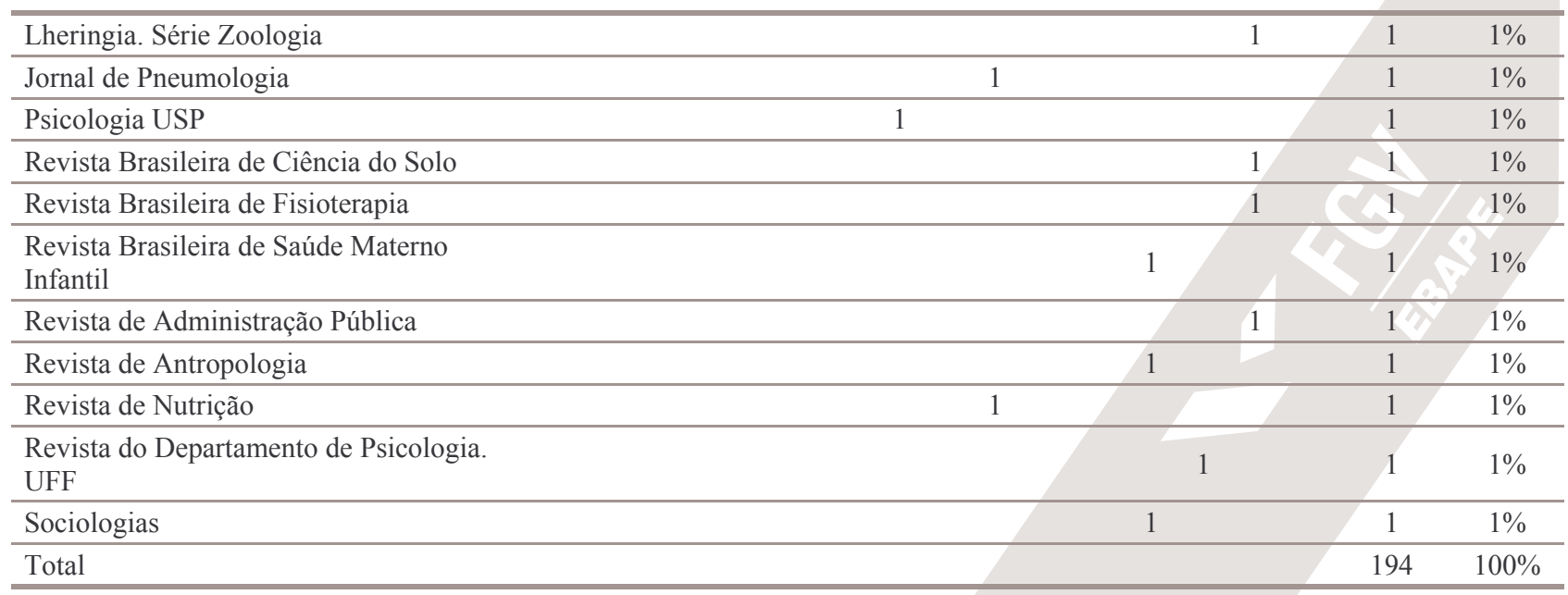

Fonte: Dados da pesquisa

Em relação à tabela 1, merecem destaque os seguintes periódicos: Reflexão e Crítica, Ciência da Informação, Revista Latino-Americana de Enfermagem, RAE eletrônica, Psicologia em Estudo e Arquivos de Neuropsiquiatria, os quais, juntos, correspondem a $41 \%$ dos artigos selecionados. Salienta-se que, a partir de 2001, o periódico Ciência da Informação apresentou, em todos os anos, artigos sobre o tema, e que o primeiro periódico a pesquisar sobre o tema foi a Revista de Saúde Pública, em 1977. Salienta-se que ao investigar a tendência dos textos, buscando verificar as ferramentas de tecnologia de informação utilizadas no âmbito organizacional no processo de aprendizagem e gestão do conhecimento, verificou-se que os artigos com esse enfoque passaram a ser publicados a partir de 2001.

Uma vez apresentados os periódicos com maior incidência do termo, buscou-se identificar a quantidade de publicações, por área, conforme classificação proposta pela Scielo em sua "lista por assunto".

Tabela 2

Agrupamento, por área, dos periódicos que apresentaram o termo aprendizagem/ gestão do conhecimento/tecnologia de informação

\begin{tabular}{lcc}
\hline Área & $\begin{array}{c}\text { No } \text { de artigos } \\
\text { onde ocorre o } \\
\text { termo }\end{array}$ & $\begin{array}{c}\text { Percentual } \\
\mathbf{( \% )}\end{array}$ \\
\hline Ciências humanas & 72 & $37 \%$ \\
\hline Ciências da saúde & 53 & $27 \%$ \\
\hline Ciências sociais aplicadas & 27 & $14 \%$ \\
\hline Engenharias & 14 & $7 \%$ \\
\hline Ciências da saúde/ciências humanas & 10 & $5 \%$ \\
\hline Ciências exatas e da Terra & 7 & $4 \%$ \\
\hline Ciências agrárias/engenharias/ciências & 4 & $2 \%$ \\
\hline humanas & 4 & $1 \%$ \\
\hline Ciências humanas/ciências sociais aplicadas & 1 & $1 \%$ \\
\hline Ciências agrárias & 1 & $1 \%$ \\
\hline Ciências biológicas & 1 & $100 \%$ \\
\hline Lingüística, letras e artes & 194 & $2 \%$ \\
\hline Total & 4 & \\
\hline
\end{tabular}

Fonte: Dados da pesquisa. 
Esse levantamento permitiu identificar que as áreas de ciências humanas, ciências da saúde e ciências sociais aplicadas, em conjunto, respondem por $78 \%$ das publicações sobre o tema. Uma vez explorados aspectos mais gerais quanto ao termo gestão do conhecimento/aprendizagem/tecnologia de informação, procedeu-se à leitura dos resumos dos artigos selecionados (194), com vistas a recortar aqueles que tratam de aspectos vinculados à gestão do conhecimento organizacional, enfocando o processo de aprendizagem organizacional e fazendo uso de ferramentas de tecnologia de informação.

\section{Segundo momento: elementos identificados na seleção de artigos}

Nesta seção, serão apresentados os seguintes itens: as instituições que pesquisaram sobre gestão do conhecimento/aprendizagem/tecnologia de informação, o número de autores que participaram da elaboração do artigo publicado e os autores que mais publicaram sobre o tema, bem como as referências mais citadas nos artigos contidos nas publicações selecionadas nesta pesquisa. Além disso, a partir da leitura e interpretação dos resumos, os artigos foram classificados quanto à aprendizagem organizacional segundo a categorização proposta por Pawlowsky, Forslin e Reinhardt (2003), sob dois enfoques: o primeiro, em relação aos processos de aprendizagem organizacional (difusão, integração, criação, ação e identificação) e o segundo quanto ao nível de aprendizagem organizacional (individual, grupo, organizacional e interorganizacional). Foram selecionados para a amostragem, os artigos que apresentaram maior número de ocorrência dos mesmos, considerando como fator excludente nessa seleção a não ocorrência de um dos elementos, seja em relação aos processos de aprendizagem, seja em relação ao nível de aprendizagem.

\section{a) Instituições de ensino dos artigos analisados}

A tabela 3 apresenta as instituições de origem dos autores das publicações científicas. No que diz respeito à vinculação institucional, a USP foi a instituição que mais apresentou publicações a respeito (20\%). Em face desse diagnóstico, pode-se inferir que a USP configura-se como a instituição benchmarking nessa área de pesquisa, uma vez que a Unicamp, segunda instituição que mais publica nessa área, é responsável por 7\% das publicações. Observa-se ainda que em relação à gestão do conhecimento organizacional, enfocando o processo de aprendizagem e fazendo uso de ferramentas de tecnologia de informação, a USP participa com 12\%, permanecendo como benchmarking nessa área de pesquisa, enquanto a PUC é a segunda instituição, respondendo por $7 \%$ das publicações. 
Investigação no contexto brasileiro sobre gestão do conhecimento/aprendizagem/tecnologia de informação: pesquisa realizada na Scientific Electronic Library Online
Wagner Igarashi Deisy Cristina Corrêa Igarashi Eleonora Milano Falcão Vieira José Leomar Todesco

Tabela 3

Instituições que pesquisaram sobre o tema da pesquisa

\begin{tabular}{|c|c|c|c|c|c|}
\hline \multirow[b]{2}{*}{$\begin{array}{l}\text { № de } \\
\text { ordem }\end{array}$} & \multirow[b]{2}{*}{ Instituições } & $\begin{array}{l}\text { Número de } \\
\text { publicações }\end{array}$ & $\begin{array}{l}\text { Percentual } \\
(\%)\end{array}$ & $\begin{array}{l}\text { Número de } \\
\text { publicações }\end{array}$ & $\begin{array}{c}\text { Percentual } \\
(\%)\end{array}$ \\
\hline & & \multicolumn{2}{|c|}{$\begin{array}{l}\text { Gestão do conhecimento/ } \\
\text { aprendizagem/ } \\
\text { tecnologia de informação }\end{array}$} & \multicolumn{2}{|c|}{$\begin{array}{l}\text { Gestão do conhecimento } \\
\text { organizacional, enfocando } \\
\text { processo de aprendizagem } \\
\text { utilizando ferramentas de } \\
\text { tecnologia de informação }\end{array}$} \\
\hline 1 & USP & 91 & $20 \%$ & 13 & $12 \%$ \\
\hline 2 & Unicamp & 31 & $7 \%$ & 4 & $4 \%$ \\
\hline 3 & PUC & 28 & $6 \%$ & 7 & $7 \%$ \\
\hline 4 & UFRJ & 28 & $6 \%$ & 2 & $2 \%$ \\
\hline 5 & UEL & 16 & $4 \%$ & 2 & $2 \%$ \\
\hline 6 & Unesp & 16 & $4 \%$ & 3 & $3 \%$ \\
\hline 7 & UFRGS & 15 & $3 \%$ & 2 & $2 \%$ \\
\hline 8 & Fiocruz & 14 & $3 \%$ & 5 & $5 \%$ \\
\hline 9 & Unifesp & 13 & $3 \%$ & 4 & $4 \%$ \\
\hline 10 & UNB & 12 & $3 \%$ & 6 & $6 \%$ \\
\hline 11 & UFMG & 9 & $2 \%$ & 4 & $4 \%$ \\
\hline 12 & UFSC & 9 & $2 \%$ & 2 & $2 \%$ \\
\hline 13 & UFSCar & 9 & $2 \%$ & 2 & $2 \%$ \\
\hline 14 & UFC & 8 & $2 \%$ & 4 & $4 \%$ \\
\hline 15 & UFF & 7 & $2 \%$ & 4 & $4 \%$ \\
\hline 16 & UFRN & (2) & $2 \%$ & 4 & $4 \%$ \\
\hline 17 & Uerj & 6 & $1 \%$ & 2 & $2 \%$ \\
\hline 18 & FGV & 5 & $1 \%$ & 2 & $2 \%$ \\
\hline 19 & Universidade do Minho(Portugal) & 5 & $1 \%$ & 1 & $1 \%$ \\
\hline \multirow[t]{2}{*}{20} & Universidade São Francisco & 5 & $1 \%$ & & $0 \%$ \\
\hline & Total & 457 & & 107 & \\
\hline
\end{tabular}

Fonte: Dados da pesquisa.

Esse levantamento permitiu identificar, ainda, a interação entre as instituições quanto à publicação conjunta. Em $81 \%$ dos artigos pesquisados, os textos foram produzidos por autores vinculados à mesma instituição; ou seja, os resultados apontam para a ausência de interação entre as instituições.

\section{b) Quantidade de autores das publicaçães cientificas}

A tabela 4 apresenta as informações referentes ao número de autores que participaram dos artigos selecionados. 
Investigação no contexto brasileiro sobre gestão do conhecimento/aprendizagem/tecnologia de informação: pesquisa realizada na Scientific Electronic Library Online
Wagner Igarashi

Deisy Cristina Corrêa Igarashi

Eleonora Milano Falcão Vieira José Leomar Todesco

\section{Tabela 4}

Número de autores participantes dos artigos selecionados

\begin{tabular}{lcccc}
\hline & Número de autores & $\begin{array}{c}\text { Número de } \\
\text { publicações }\end{array}$ & Número de autores & $\begin{array}{c}\text { Número de } \\
\text { publicações }\end{array}$ \\
\cline { 2 - 5 } & $\begin{array}{c}\text { Gestão do conhecimento/aprendizagem/ } \\
\text { tecnologia de informação }\end{array}$ & $\begin{array}{c}\text { Gestão do conhecimento organizacional, } \\
\text { enfocando o processo de aprendizagem } \\
\text { e fazendo uso de ferramentas de } \\
\text { tecnologia de informação }\end{array}$ \\
\hline 1 autor & 59 & $30 \%$ & 14 & $29 \%$ \\
\hline 2 autores & 66 & $34 \%$ & 18 & $37 \%$ \\
\hline 3 autores & 35 & $18 \%$ & 11 & $22 \%$ \\
\hline Mais de 3 autores & 34 & $18 \%$ & 6 & $12 \%$ \\
\hline Total & 194 & $100 \%$ & 49 & $100 \%$ \\
\hline
\end{tabular}

Fonte: Dados da pesquisa.

A tabela 4 mostra que $30 \%$ dos artigos pesquisados tiveram apenas um autor, que $34 \%$ resultaram de co-autoria com a participação de dois autores e que em $18 \%$ dos casos houve co-autoria envolvendo três ou mais de três autores. Quando analisados os artigos sobre gestão do conhecimento organizacional, enfocando o processo de aprendizagem e utilizando ferramentas de tecnologia de informação, verificou-se que $29 \%$ dos artigos tiveram apenas um autor, que $37 \%$ deles tiveram co-autoria com a participação de dois autores, que em $22 \%$ dos casos a co-autoria envolveu a participação de três autores e em $12 \%$, de mais de três autores.

\section{c) Autores que mais publicaram sobre tema}

A tabela 5 apresenta os 13 autores que mais publicaram nos periódicos a respeito do assunto gestão do conhecimento/aprendizagem/tecnologia de informação no período de 1977 a 2007.

\section{Tabela 5}

Autores que mais publicaram sobre o assunto

\begin{tabular}{lcc}
\hline Autor & $\begin{array}{c}\text { No de artigos } \\
\text { publicados } \\
\text { pelos autores }\end{array}$ & Percentual (\%) \\
\hline Maria Beatriz Martins Linhares & 8 & $4 \%$ \\
\hline Edna Maria Marturano & 6 & $3 \%$ \\
\hline Sônia Regina Loureiro & 5 & $3 \%$ \\
\hline Evely Boruchovitch, Fermino Fernandes Sisto, Sérgio Luís da Silva & 4 & $2 \%$ \\
\hline $\begin{array}{l}\text { Dejano T. Sobral, Marlo Cunha, Maurício Cagy, Pedro Ribeiro, } \\
\text { Roberto Piedade, Vernon Furtado e Victor Hugo Bastos }\end{array}$ & 3 & $2 \%$ \\
\hline
\end{tabular}

Fonte: Dados da pesquisa.

A pesquisa demonstrou a inexistência de pesquisadores brasileiros com trajetória estabelecida na área de gestão do conhecimento/aprendizagem/tecnologia de informação, conforme a amostragem do período investigado (1977-2007). Quando se limita esta pesquisa à amostragem da gestão do conhecimento organizacional, enfocando o processo de aprendizagem e fazendo uso de ferramentas de tecnologia de informação, o quadro se torna precário. Apenas três autores, Sérgio Luís da Silva, Roberto Carlos dos Santos Pacheco e Vinícius 
Investigação no contexto brasileiro sobre gestão do conhecimento/aprendizagem/tecnologia de informação: pesquisa realizada na Scientific Electronic Library Online
Wagner Igarashi

Deisy Cristina Corrêa Igarashi

Eleonora Milano Falcão Vieira José Leomar Todesco

Medina Kern, apresentam, cada um, dois artigos publicados na área, o que corresponde a 4\% do total de artigos (amostragem composta por 49 artigos).

Além disso, esse levantamento permitiu identificar que os 194 artigos contaram com a participação de 469 autores e que os 49 artigos selecionados na amostragem contaram com 106 autores. Conforme a tabela 5, percebe-se que apenas 13 autores publicaram mais de três trabalhos acerca do tema. Outra preocupação deste estudo foi em relação a referências adotadas pelos pesquisadores para o desenvolvimento dos artigos, conforme apresentado a seguir.

\section{d) Referência bibliográfica}

De um universo de 4.886 referências bibliográficas, constantes nos 194 artigos selecionados, foram encontradas 714 referências relativas à gestão do conhecimento organizacional, enfocando o processo de aprendizagem e fazendo uso de ferramentas de tecnologia de informação. A tabela 6 seleciona as referências citadas em cinco ou mais artigos dos 49 da amostragem.

\section{Tabela 6}

Referências mais citadas nos artigos sobre gestão do conhecimento organizacional, enfocando o processo de aprendizagem e fazendo uso de ferramentas de tecnologia de informação

\begin{tabular}{ccc}
\hline No de ordem & Autores referenciados & No de vezes \\
\hline 1 & NONAKA, I; TAKEUCHI, H. & 22 \\
\hline 2 & VYGOTSKY, L. S. & 17 \\
\hline 3 & SENGE, P. & 13 \\
\hline 4 & SISTO, F. F. & 13 \\
\hline 5 & ZIMMERMAN, B. J. & 11 \\
\hline 6 & PORTER, M. & 8 \\
\hline 7 & YIN, R. & 8 \\
\hline 8 & CASTELLS, M. A & 7 \\
\hline 9 & MARLER PND PATERS S. & 7 \\
\hline 10 & MEZIROW, J. & 7 \\
\hline 11 & SKINNERr, B. F. & 7 \\
\hline 12 & STEWART, T. A. & 7 \\
\hline 13 & SVEIBY, K. E. & 6 \\
\hline 14 & EDVINSSON, L. & 6 \\
\hline 15 & MINTZBERG, H. & 6 \\
\hline 16 & VIANNA, M. R. & 6 \\
\hline 17 & WIIG, K. M. & 5 \\
\hline 18 & ARGYRIS, C. & 5 \\
\hline 19 & SPENDER, J. C. & \\
\hline
\end{tabular}

Fonte: Dados da pesquisa.

Entre as referências mais citadas foi observada que as citações a EDVINSSON, NONAKA, SENGE, STEWART, SVEIBY e a TAKEUCHI referem-se a obras (livros) traduzidas, publicadas no país na década de 1990, mais especificamente entre 1995 e 1998.

Uma vez apresentadas as informações extraídas dos artigos de ordem geral, passa-se a apresentar informações mais específicas, a partir da leitura e análise dos 49 artigos selecionados, relativos à gestão do conhecimento organizacional, enfocando o processo de aprendizagem e fazendo uso de ferramentas de tecnologia de 
Investigação no contexto brasileiro sobre gestão do conhecimento/aprendizagem/tecnologia de informação: pesquisa realizada na Scientific Electronic Library Online
Wagner Igarashi

Deisy Cristina Corrêa Igarashi Eleonora Milano Falcão Vieira José Leomar Todesco

informação, com vistas a: (i) a categorizar os artigos segundo a percepção de Pawlowsky, Forslin e Reinhardt (2003), quanto ao processo e ao nível de aprendizagem organizacional.

\section{e) Categorização dos artigos quanto ao processo e ao nível de aprendizagem organizacional}

A tabela 7 apresenta a categorização dos artigos selecionados, segundo a percepção de Pawlowsky, Forsline Reinhardt (2003) quanto à aprendizagem, a qual pode ser vista segundo dois enfoques. O primeiro, em relação aos processos de aprendizagem organizacional, está sujeito a cinco categorizações:

- difusão - considera relevante o processo de troca e distribuição de conhecimento;

- integração - observa questões referentes à manutenção, ao armazenamento e à segurança do conhecimento organizacional;

- criação - refere-se à fase do aprendizado organizacional focada na criação de conhecimento, no desenvolvimento de novas idéias e na inovação;

- ação - analisa as conseqüências ambientais do aprendizado e quais procedimentos traduzem novos desenvolvimentos em ação; e

- identificação - aborda questões relacionadas à avaliação da aprendizagem, para facilitar a identificação interna e externa do conhecimento.

- Quanto ao segundo enfoque, este considera o nível de aprendizagem organizacional e pode ser segmentado em quatro categorizações:

- individual - que considera o conhecimento por indivíduo que atua junto à organização;

- grupo - enfoca a interação, quanto à aprendizagem, entre grupos da organização;

- organizacional - que observa a aprendizagem em toda a organização; e

- interorganizacional - verifica-se que aprendizagem organizacional extrapola o ambiente interno, gerando reflexo nos segmentos com os quais a organização interage.

Considerando as características dos dois enfoques, procedeu-se à leitura dos 49 artigos, com vistas a verificar e identificar esses elementos nos textos. Os resultados observados são apresentados na tabela 7.

A partir do número de elementos identificados, optou-se por selecionar, a partir da amostragem, os artigos que apresentaram maior número de ocorrência dos mesmos. Nesse estudo, foi definido como fator excludente para a seleção dos artigos, a não ocorrência de um dos elementos, seja em relação ao processo ou em relação ao nível. Ao considerar essa delimitação, foram selecionados oito artigos, os quais são detalhados na seção seguinte. 
Investigação no contexto brasileiro sobre gestão do conhecimento/aprendizagem/tecnologia de informação: pesquisa realizada na Scientific Electronic Library Online
Wagner Igarashi

Deisy Cristina Corrêa Igarashi

Eleonora Milano Falcão Vieira José Leomar Todesco

Tabela 7

Artigos classificados quanto a abordagem, natureza e ótica

\begin{tabular}{ccc}
\hline & $\begin{array}{c}\text { Número de } \\
\text { artigos } \\
\text { identificados }\end{array}$ & Percentual \% \\
\hline Todos os 9 elementos & 1 & $2 \%$ \\
\hline 8 elementos & 3 & $6 \%$ \\
\hline 7 elementos & 3 & $6 \%$ \\
\hline 6 elementos & 3 & $6 \%$ \\
\hline 5 elementos & 4 & $8 \%$ \\
\hline 4 elementos & 9 & $18 \%$ \\
\hline 3 elementos ou menos & 26 & $53 \%$ \\
\hline Total de artigos & 49 & $100 \%$ \\
\hline
\end{tabular}

Fonte: Dados da pesquisa.

Terceiro momento: gestão do conhecimento organizacional, enfocando o processo de aprendizagem, segundo a classificação de Pawlowsky, Forslin e Reinhardt (2003) e fazendo uso de ferramentas de tecnologia de informação

Esta subseção apresenta um mapeamento feito nas publicações da amostra (oito artigos) referentes à gestão do conhecimento, enfocando o processo de aprendizagem organizacional e fazendo uso de ferramentas de tecnologia de informação. São considerados os artigos que apresentaram maior número de elementos segundo a classificação de Pawlowsky, Forslin e Reinhardt (2003). O quadro 1 apresenta as informações resultantes da análise, de modo cronológico. 


\section{Quadro 1}

Tendências emergentes em estudos nacionais voltados a gestão do conhecimento organizacional, enfocando o processo de aprendizagem e fazendo uso de ferramentas de tecnologia de informação

\begin{tabular}{|c|c|c|c|}
\hline Autor & $\begin{array}{l}\text { Aspectos } \\
\text { abordados no } \\
\text { artigo }\end{array}$ & Enfoque do artigo & Tendências observadas \\
\hline 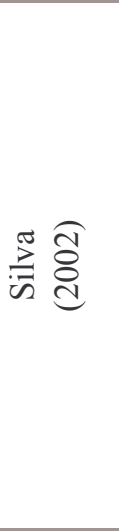 & $\begin{array}{l}\text { Discute elementos } \\
\text { de informação, } \\
\text { competitividade, } \\
\text { gestão do } \\
\text { conhecimento, } \\
\text { processos } \\
\text { organizacionais e } \\
\text { tecnologia da } \\
\text { informação }\end{array}$ & $\begin{array}{l}\text { Teórico - analisa a } \\
\text { gestão do } \\
\text { conhecimento em três } \\
\text { níveis: estratégico, } \\
\text { tático e operacional. } \\
\text { Nesse último descreve } \\
\text { a questão da } \\
\text { aprendizagem, os } \\
\text { formatos do } \\
\text { conhecimento e o papel } \\
\text { da tecnologia da } \\
\text { informação }\end{array}$ & $\begin{array}{l}\text { Aborda a gestão do conhecimento por meio de } \\
\text { processos organizacionais orientados pelo } \\
\text { conhecimento, a gestão por competências; a } \\
\text { qualidade enfoca os processos } \\
\text { interorganizacionais; busca de conhecimentos } \\
\text { externos (benchmarking de melhores práticas); } \\
\text { utilização de novas ferramentas de tecnologia de } \\
\text { informação para apoiar a gestão do } \\
\text { conhecimento; criação de novos espaços para } \\
\text { conversão de conhecimento tácito/explícito na } \\
\text { produção; marketing com pesquisas externas e } \\
\text { criação de espaços para incorporação de } \\
\text { conhecimentos de clientes externos. }\end{array}$ \\
\hline 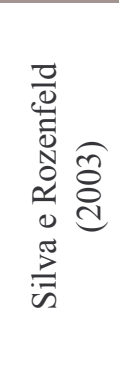 & $\begin{array}{l}\text { Avalia a gestão do } \\
\text { conhecimento, } \\
\text { conversões de } \\
\text { conhecimento e } \\
\text { desenvolvimento de } \\
\text { produto }\end{array}$ & $\begin{array}{l}\text { Empírico - apresenta a } \\
\text { utilização de um } \\
\text { modelo de avaliação da } \\
\text { gestão do } \\
\text { conhecimento, } \\
\text { enfocando o processo } \\
\text { de desenvolvimento de } \\
\text { produtos no setor } \\
\text { automotivo }\end{array}$ & $\begin{array}{l}\text { Menciona os modelos de avaliação da gestão de } \\
\text { conhecimento, sinalizando lacunas existentes. } \\
\text { Contudo, não detalha os passos para promover as } \\
\text { melhorias, nem as tecnologias de informação } \\
\text { adotadas, pois, segundo os autores, estes devem } \\
\text { ser explorados conforme o contexto } \\
\text { organizacional. Além disso, é apresentada uma } \\
\text { visão da gestão de conhecimento sob a ótica do } \\
\text { desenvolvimento de produtos. }\end{array}$ \\
\hline 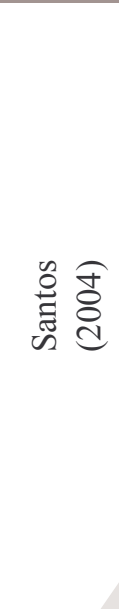 & $\begin{array}{l}\text { Explana aspectos } \\
\text { teóricos de gestão de } \\
\text { recursos humanos e } \\
\text { as práticas adotadas }\end{array}$ & $\begin{array}{l}\text { Teórico - apresenta o } \\
\text { consenso das vias que } \\
\text { podem ser utilizadas } \\
\text { para o desenvolvimento } \\
\text { do capital intelectual de } \\
\text { uma organização } \\
\text { (humano, estrutural e } \\
\text { relacional) e discute a } \\
\text { questão da } \\
\text { segmentação dos } \\
\text { recursos humanos }\end{array}$ & $\begin{array}{l}\text { Considera que a tecnologia de informação } \\
\text { desencadeou a necessidade de flexibilização das } \\
\text { organizações, fazendo com que se tornasse } \\
\text { necessário o desenvolvimento do capital } \\
\text { humano, bem como da segmentação de seus } \\
\text { recursos humanos (facilmente substituíveis e } \\
\text { com baixo valor agregado à empresa; facilmente } \\
\text { substituíveis e com elevado valor agregado; } \\
\text { difíceis de substituir, mas com baixo valor } \\
\text { agregado e difíceis de substituir, com alto valor } \\
\text { agregado). Considera que as estratégias de } \\
\text { desenvolvimento de capital intelectual têm sido } \\
\text { orientadas para as atividades e recursos } \\
\text { diretamente relacionados à atividade principal da } \\
\text { organização }\end{array}$ \\
\hline
\end{tabular}


Teórico - apresenta reflexões sobre foresight, inteligência

Aborda a gestão da inovação competitiva e gestão do conhecimento, para o aperfeiçoamento conceitual da gestão da inovação

Enfoca a necessidade de incremento do processo de tomada de decisão e os efeitos de networking, como chave para a promoção da capacidade de inovação e competitividade. Considera o processo permanente de produção de conhecimento e decisões como eventos discretos que emergem em momentos específicos. Observam que a organização em rede possui características como horizontalidade, responsabilidades compartilhadas, procedimentos interativos de decisão e parcerias com diversos atores externos, sendo a tomada de decisão baseada em possibilidades tecnológicas de futuro.

Observa que as redes sociais influenciam no compartilhamento de informações e do conhecimento de pessoas envolvidas numa temática específica ou com interesses comuns, oportunizando o desenvolvimento de inovações. Melhor eficiência através de ferramentas que permitam uma postura cooperativa. Necessidade de canais que permitam a comunicação visual e textual. O direcionamento dos fluxos dessas redes pode fortalecer e delinear a rede

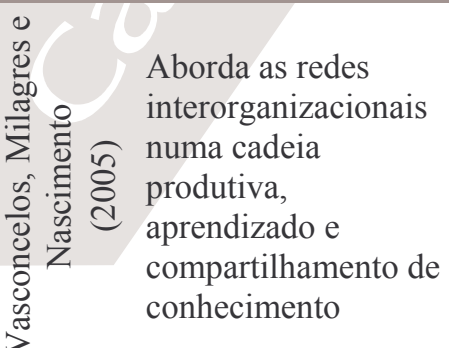

Empírico - discute a relação sobre aprendizagem organizacional e as redes em que estão inseridos, na promoção da inovação Considera que os produtos obtêm seu valor Empírico - análise das relações interorganizacionais dentro da cadeia de valores no setor automotivo, com foco na aprendizagem e no compartilhamento de conhecimento através de redes interorganizacionais. Observa que no setor automobilístico brasileiro há indícios de que exista espaço para a melhoria dessas relações, mesmo no contexto competitivo em que já se encontram, sendo necessária uma visão sistêmica da cadeia produtiva, onde a melhoria em cada elemento da cadeia produtiva poderá agregar mais valor aos produtos finais

Aborda a gestão de conhecimento enfocando que o conhecimento pode ser explicitado por meio de ferramentas de tecnologia de informação. Aplica um modelo para mapeamento de falhas, apresentado num sistema de informação através de um software de mapeamento de falhas que sistematização para o mapeamento de falhas por meio de um sistema mostrou-se apto à gestão do conhecimento. de informação no setor de energia elétrica

Considera que a externalização do conhecimento para a criação de sistemas pode estimular a troca e a conversão de conhecimento, podendo ainda ser facilmente disseminada através de uma intranet 


\begin{tabular}{|c|c|c|}
\hline $\begin{array}{l}\text { Aborda a } \\
\text { aprendizagem } \\
\text { corporativa e } \\
\text { métricas para a } \\
\text { acumulação de } \\
\text { capacidades } \\
\text { tecnológicas }\end{array}$ & $\begin{array}{l}\text { Empírico - estudo em } \\
\text { uma empresa de } \\
\text { transmissão de energia } \\
\text { elétrica no Norte do } \\
\text { Brasil; analisa sua } \\
\text { trajetória em relação } \\
\text { aos processos de } \\
\text { aprendizagem } \\
\text { corporativa para a } \\
\text { acumulação } \\
\text { tecnológica }\end{array}$ & $\begin{array}{l}\text { Considera que a aprendizagem para a } \\
\text { acumulação de capacidades tecnológicas no nível } \\
\text { individual não era convertida para o nível } \\
\text { organizacional. Observam que os cursos em } \\
\text { língua estrangeira permitiram um novo canal de } \\
\text { comunicação externo e que a criação de } \\
\text { processos de codificação de conhecimento } \\
\text { facilitou a disseminação da aprendizagem no } \\
\text { nível organizacional. Destacam que, embora } \\
\text { aplicando as métricas de capacidade tecnológica } \\
\text { e aprendizagem a um tipo de empresa ainda não } \\
\text { examinada pela literatura relacionada, o estudo } \\
\text { identificou a gestão dos diversos processos } \\
\text { internos de aprendizagem como variável-chave } \\
\text { para a maneira e a velocidade com que a empresa } \\
\text { acumula suas capacidades tecnológicas }\end{array}$ \\
\hline
\end{tabular}

Fonte: Dados da pesquisa.

Em relação aos textos selecionados (quadro 1), verifica-se que três estudos enfocavam apenas aspectos teóricos, ao passo que cinco, além da revisão conceitual, apresentam estudos empíricos (estudos de caso, análise de contexto organizacional, entrevistas e questionários, entre outras técnicas aplicadas). Destaca-se que nos dois momentos observou-se que a tecnologia de informação é considerada, buscando apoiar esse processo. Tal observação corrobora este estudo, à medida que confirma a percepção dos autores de que

a gestão do conhecimento organizacional refere-se a um processo de mudança cultural, sendo a aprendizagem um elemento essencial, que deveria ser apoiado por ferramentas de tecnologia de informação.

Para ilustrar essa afirmação a partir da releitura dos textos, buscou-se extrair elementos que sinalizassem a correlação entre a percepção dos autores que compuseram a amostragem e os autores deste estudo. Em um primeiro momento, foram resgatados os elementos relativos aos três estudos teóricos. Santos (2004) considera que

a tecnologia de informação desencadeou a necessidade de flexibilização das organizações, fazendo com que se tornasse necessário o desenvolvimento do capital humano, bem como a segmentação de seus recursos humanos.

A partir da observação de Santos (2004), pode-se inferir que ele considera a tecnologia de informação uma ferramenta que alavanca o processo de aprendizagem, uma vez que ela se refere a um dos elementos que compõem o capital humano, segundo Stewart (1998) e Sveiby (1998). Entretanto o Santos (2004) não faz menção ao apoio que a tecnologia de informação pode oferecer nesse processo. Essa lacuna é preenchida por meio da observação de Silva (2002), o qual considera a "utilização de novas ferramentas de tecnologia de informação para apoiar a gestão do conhecimento". Desse modo, a partir da percepção de Santos (2004) e de Silva (2002), verifica-se a possibilidade da tecnologia de informação ser, num primeiro momento, a ferramenta que alavanca esse processo e, num segundo momento, ser a ferramenta que auxilia esse processo, respectivamente. Nesse sentido, a percepção de Canongia et al (2004) consolida os entendimentos anteriores, pois, para eles "organização em rede possui características como horizontalidade, responsabilidades compartilhadas, procedimentos interativos de decisão e parcerias com diversos atores externos, sendo a tomada de decisão baseada em possibilidades tecnológicas de futuro". Para Canongia et al (2004), a tecnologia de informação auxilia o processo de gestão de conhecimento como um todo (alavanca, conduz e direciona), do qual faz parte a aprendizagem organizacional.

Em relação aos elementos destacados quanto aos cinco estudos empíricos, verifica-se também a possibilidade da tecnologia de informação auxiliar no processo de gestão de conhecimento como um todo (alavanca, conduz 
Investigação no contexto brasileiro sobre gestão do conhecimento/aprendizagem/tecnologia de informação: pesquisa realizada na Scientific Electronic Library Online
Wagner Igarashi

Deisy Cristina Corrêa Igarashi

Eleonora Milano Falcão Vieira José Leomar Todesco

e direciona). Para ilustrar esse entendimento, foram resgatadas as observações de Almeida et al (2006), os quais consideram "a gestão de conhecimento, enfocando que o conhecimento pode ser explicitado por meio de ferramentas de tecnologia de informação". Dessa afirmação, pode-se inferir que a tecnologia de informação estaria apoiando o processo de alavancagem da gestão do conhecimento.

Em um segundo momento, o mesmo autor observa que aplicou "um modelo para mapeamento de falhas, apresentado em um sistema de informação através de um software de mapeamento de falhas que se mostrou apto à Gestão do Conhecimento". Nesse segundo momento, observa-se que a tecnologia de informação alicerça o processo que conduz à gestão do conhecimento.

Finalmente, em um terceiro momento, o mesmo autor salienta que a "externalização do conhecimento para a criação de sistemas pode estimular a troca, a conversão de conhecimento e pode facilmente ser disseminada através de uma intranet"; ou seja, nesse caso, a tecnologia de informação estaria direcionando o processo de gestão do conhecimento. Observa-se que esses momentos (alavancagem, condução e direcionamento) também são observados nos outros estudos empíricos (VASCONCELOS; MILAGRES; NASCIMENTO, 2005; ROSA; FIGUEIREDO, 2006; SILVA; ROZENFELD, 2003; TOMAÉL; ALCARÁ; CHIARA, 2005), os quais podem ser visualizados no quadro 1.

Além disso, merece destaque o entendimento de Silva e Rozenfeld (2003), os quais "não detalham os passos para promover as melhorias, nem as tecnologias de informação adotadas, pois segundo os autores estes devem ser explorados conforme o contexto organizacional". Esse entendimento proporciona um alerta aos pesquisadores que enfocam a gestão do conhecimento/aprendizagem/tecnologia de informação, em relação à necessidade de adaptar a(s) ferramenta(s) selecionada(s) ao contexto organizacional, observando aspectos intrínsecos, tais como sua cultura e valores.

A partir das percepções, os autores do estudo acreditam ser viável a proposição de um modelo, buscando avaliar a aprendizagem organizacional. Assim, seriam gerados subsídios para a gestão do conhecimento, calcados na tecnologia de informação, desde que observada a preocupação de Silva e Rozenfeld (2003) quanto às características intrínsecas a uma organização.

Ainda quanto à proposição do modelo, os estudos selecionados consideram algumas lacunas que devem ser observadas. Silva, Rozenfeld (2003), por exemplo, observam que "os modelos de avaliação da gestão de conhecimento permitem sinalizar lacunas existentes. Contudo, não detalham os passos para promover as melhorias". Rosal e Figueiredo (2006) consideram que "um problema da aprendizagem organizacional é que grande parte do conhecimento no nível individual não é convertido para o nível organizacional". Outro aspecto, observado por Vasconcelos, Milagres, Nascimento (2005) e por Silva (2002), refere-se à importância do compartilhamento de conhecimento no nível interorganizacional na agregação e criação de produtos inovadores. No entanto, conforme observado por Vasconcelos, Milagres, Nascimento (2005) no setor estudado por eles, o automobilístico, "há espaço para a evolução das relações interorganizacionais". Em relação a essa última observação, poderiam ser analisados setores diferenciados para verificar se também existe a mesma necessidade.

\section{Considerações finais}

Para o alcance do objetivo deste estudo, a investigação foi conduzida em três momentos. Inicialmente, esta pesquisa focalizou 194 trabalhos que abordavam os termos gestão do conhecimento/aprendizagem/tecnologia de informação, com vistas a identificar os periódicos em que os artigos foram publicados e as áreas de conhecimento que mais têm publicado sobre o tema. Como resultado, identificou-se que Reflexão e Crítica, Ciência da Informação, Revista Latino-Americana de Enfermagem, RAE eletrônica, Psicologia em Estudo e Arquivos de Neuropsiquiatria foram os periódicos com maior ocorrência dos termos. Na seqüência, esta pesquisa delimitou a amostra, selecionando os trabalhos que tratam de aspectos relacionados à gestão do conhecimento organizacional, enfocando o processo de aprendizagem e fazendo uso de ferramentas de 
Investigação no contexto brasileiro sobre gestão do conhecimento/aprendizagem/tecnologia de informação: pesquisa realizada na Scientific Electronic Library Online
Wagner Igarashi

Deisy Cristina Corrêa Igarashi

Eleonora Milano Falcão Vieira José Leomar Todesco

tecnologia de informação. Assim, procedeu-se à leitura dos resumos, com vistas a selecionar aqueles que tratam do tema.

Essa seleção resultou em 49 artigos e o estudo bibliométrico apresentou os seguintes resultados: a maioria dos artigos foi de autores afiliados à USP (12\%) e as publicações foram produzidas por autores vinculados a uma mesma instituição, o que demonstra a inexistência de interação entre as instituições quanto à publicação conjunta. Verificou-se no tema gestão do conhecimento organizacional, enfocando o processo de aprendizagem e fazendo uso de ferramentas de tecnologia de informação, que não existe no contexto nacional autores consolidados, destacando-se apenas três autores com duas publicações sobre tema. Verificou-se também quanto às referências adotadas de livros traduzidos: Edvinsson, Nonaka, Senge, Stewart, Sveiby e Takeuchi.

Destaca-se que, para selecionar os artigos explorados no terceiro momento da pesquisa, foi adotada a categorização proposta por Pawlowsky, Forslin e Reinhardt (2003) quanto à aprendizagem organizacional, a qual pode ser segmentada sob dois enfoques: o primeiro, em relação aos processos de aprendizagem organizacional (difusão, integração, criação, ação e identificação) e o segundo quanto ao nível de aprendizagem organizacional (individual, grupo, organizacional e interorganizacional). Foram selecionados para a amostragem, os artigos que apresentaram maior número de ocorrência dos mesmos, considerando como fator excludente para a seleção dos artigos a não ocorrência de um dos elementos, seja em relação aos processos de aprendizagem ou em relação ao nível de aprendizagem.

Esse mapeamento levou à identificação de enfoques e percepções que permitiram verificar que o entendimento dos autores quanto à gestão do conhecimento organizacional se refere a um processo de mudança cultural, sendo a aprendizagem um elemento essencial, que deveria ser apoiado por ferramentas de tecnologia de informação, permitindo ainda agregar a esse entendimento, dois novos elementos, a saber: (i) a possibilidade da tecnologia de informação auxiliar no processo de gestão de conhecimento como um todo (alavancagem, condução e direcionamento), e (ii) a necessidade de adaptar a ferramenta selecionada ao contexto organizacional, observando aspectos intrínsecos, tais como sua cultura e valores, entre outros.

Como uma limitação da pesquisa, destaca-se que o estudo foi desenvolvido utilizando a percepção no contexto nacional, restrito apenas ao banco de dados relativo à biblioteca eletrônica Scientific Electronic Library Online (Scielo). Por isso, os indicadores gerados não podem ser considerados em contexto mais amplo.

A partir da limitação apontada, como tópico para futura pesquisa, recomenda-se que seja realizada busca similar em outras bases de dados - tais como os periódicos com conceito "A, B e C" e anais de congresso reconhecidos pelo sistema Qualis/Capes - e que também seja ampliada, de modo a considerar o contexto internacional. Além disso, a pesquisa pode ser enriquecida com a busca por outros indicadores.

Além das recomendações de concepção teórica, existe também a possibilidade de se construir um modelo para avaliar a aprendizagem organizacional, gerando assim subsídios para a gestão do conhecimento, calcados na tecnologia de informação, uma vez observadas as características intrínsecas a uma organização. 
Investigação no contexto brasileiro sobre gestão do conhecimento/aprendizagem/tecnologia de informação: pesquisa realizada na Scientific Electronic Library Online
Wagner Igarashi

Deisy Cristina Corrêa Igarashi

Eleonora Milano Falcão Vieira José Leomar Todesco

\section{Referências}

ALMEIDA, Dagoberto Alves de et al. Gestão do conhecimento na análise de falhas: mapeamento de falhas através de sistema de informação. Prod., São Paulo, v.16, n.1, 2006. Disponivel em: <http://www.scielo.br/scielo.php?script=sci_arttextCtpid=S010365132006000100014 CtIng=enctnrm=iso>. Acesso em: 14 de maio 2007.

BITENCOURT, Cláudia. A gestão de competências como alternativa de formação e desenvolvimento nas organizações: uma reflexão crítica baseada na percepção de um grupo de gestores. In: RUAS, Roberto Lima; ANTONELLO, Claudia Simone; BOFF, Luiz Henrique (Org.). Os novos horizontes da gestão: aprendizagem organizacional e competências. Porto Alegre: Bookman, 2005. p.132-149.

CALDAS, M. P.; TINOCO, T.; CHU, R. A. Análise bibliométrica dos artigos de RH publicados no ENANPAD na década de 1990: um mapeamento a partir das citações dos heróis, endogenias e jactâncias que fizeram a história recente da produção científica da área. In: ENANPAD, 27., 2003, Atibaia-SP. Anais... Rio de Janeiro: ANPAD, 2003. CD-ROM.

CANONGIA, Claudia et al. Foresight, inteligência competitiva e gestão do conhecimento: instrumentos para a gestão da inovação. Gestão At Produção, São Carlos, v.11, n.2, 2004. Disponível em: <http://www.scielo.br/scielo.php?script=sci_arttextEtpid=S0104530X2004000200009\&t|ng=enctnrm=iso >. Acesso em: 14 de maio 2007.

FIALHO, Francisco Antônio Pereira, et al. Gestão do conhecimento e aprendizagem: as estratégias competitivas da sociedade pósindustrial. Florianópolis: Visual Books 2006.

LEITE, João Batista Diniz; PORSSE, Melody de Campos Soares. Gestão por competências e aprendizagem organizacional: em busca da vantagem competitiva.. In: RUAS, Roberto Lima; ANTONELLO, Claudia Simone; BOFF, Luiz Henrique (Org.). Os novos horizontes da gestão: aprendizagem organizacional e competências. Porto Alegre: Bookman, 2005. p.56-69.

MACIAS-CHAPULA, C. A. 0 papel da informetria e da cienciometria e sua perspectiva nacional e internacional. Ciência da Informação, Brasilia, v.27, n.2, p.134-140, maio/ago. 1998.

PAWLOWSKY, P.; FORSLIN, J.; REINHARDT, R. Practices and tools of organizational learning. In: DIERKES, M.; ANTAL, A. B.; CHILD, J.; NONAKA, I. Handbook of Organizational Learning and Knowledge. Oxford: Oxford, p.775-793, 2003.

PRITCHARD, C. Trends in economic evaluation. OHE Briefing, n. 36, abr. 1998.

ROSAL, Anna Carolina Lemos; FIGUEIREDO, Paulo Negreiros. Aprendizagem corporativa e acumulação tecnológica: a trajetória de uma empresa de transmissão de energia elétrica no Norte do Brasil. Gestão \&t Produção, São Carlos, v.13, n.1, 2006. Disponivel em: <http://www.scielo.br/scielo.php?script=sci_arttext\&tpid=S0104-530X2006000100004Ctlng=en\&tnrm=iso>. Acesso em: 14 de maio 2007.

RUAS, Roberto Lima. Gestão por competências: uma contribuição à estratégia das organizações. In: RUAS, Roberto Lima; ANTONELLO, Claudia Simone; BOFF, Luiz Henrique (Org.). Os novos horizontes da gestão: aprendizagem organizacional e competências. Porto Alegre: Bookman, 2005. p.34-55.

SANTOS, Maria João Nicolau. Gestão de recursos humanos: teorias e práticas. Sociologias, Porto Alegre, n.12, 2004. Disponível em: <http://www.scielo.br/scielo.php?script=sci_arttext\&tpid=S1517-45222004000200006CtIng=en\&tnrm=iso>. Acesso em: 14 maio 2007.

SIELO. Scientific Electronic Library Online, 2007. Disponível em: <http:// http://www.scielo.org>. Acesso em: 14 de maio 2007.

SILVA, Sérgio Luis da. Informação e competitividade: a contextualização da gestão do conhecimento nos processos organizacionais. Ciência da Informação, Brasilia, v.31, n.2, 2002. Disponivel em: <http://www.scielo.br/scielo.php?script=sci_arttext\&tpid=S0100$19652002000200015 \&$ tlng=ptCtnrm=iso>. Acesso em: 14 de maio 2007.

SILVA, Sérgio Luis da; ROZENFELD, Henrique. Modelo de avaliação da gestão do conhecimento no processo de desenvolvimento do produto: aplicação em um estudo de caso. Prod., São Paulo, v.13, n.2, 2003. Disponível em: <http://www.scielo.br/scielo.php?script=sci_arttext\&tpid=S0103-65132003000200002氏ting=en\&tnrm=iso>. Acesso em: 14 de maio 2007.

STEWART, Thomas. A. Capital intelectual: a nova vantagem competitiva das empresas. Rio de Janeiro: Campus, 1998.

SVEIBY, Karl Erik. A nova riqueza das organizações. Rio de Janeiro: Campus, 1998.

TOMAEL, Maria Inês; ALCARA, Adriana Rosecler; DI CHIARA, Ivone Guerreiro. Das redes sociais à inovação. Ciência da Informação, Brasilia, v.34, n.2, 2005. Disponivel em: <http://www.scielo.br/scielo.php?script=sci_arttextctpid=S0100-19652005000200010\&t|ng=en\&tnrm=iso >. Acesso em: 14 de maio 2007. 
Investigação no contexto brasileiro sobre gestão do conhecimento/aprendizagem/tecnologia de informação: pesquisa realizada na Scientific Electronic Library Online
Wagner Igarashi

Deisy Cristina Corrêa Igarashi

Eleonora Milano Falcão Vieira José Leomar Todesco

VASCONCELOS, Maria Celeste Reis Lobo; MILAGRES, Rosiléia; NASCIMENTO, Edna do. Estratégia de relacionamento entre os membros da cadeia produtiva no Brasil: reflexões sobre o tema. Gestão \& Produção, São Carlos, v.12, n.3, 2005. Disponivel em: $<$ <ttp://www.scielo.br/scielo.php?script=sci_arttext\&tpid=S0104-530X2005000300009\&tlng=en\&tnrm=iso>. Acesso em: 14 de maio 2007. 